CARTA AL DIRECTOR

\title{
Hospitalización parcial y evolución de la anorexia nerviosa
}

\author{
C. Rodríguez ${ }^{a}$, B. Fernández-Corres ${ }^{a}$, M.J. Pérez ${ }^{b}$ A. Iruin ${ }^{b}$ A. González Pinto \\ -Departamento de Psiquiatría, Hospital Santiago Apóstol, Olaguibel 29, 01004, Vitoria, España; 'Sistema de \\ Salud Mental Osakidetza, Vitoria, España.
}

\section{Sr. Director:}

Hay mucho debate en la actualidad sobre las mejores estructuras de recursos para el tratamiento de los pacientes psiquiátricos graves. La hospitalización parcial, una modalidad de tratamiento no residencial, ha disfrutado de popularidad renovada. Hay un debate considerable sobre los programas comunitarios relacionados con los pacientes psicóticos. Sin embargo, el uso de la hospitalización parcial es común también en otros trastornos como la anorexia nerviosa [1]. Aunque la hospitalización parcial no es adecuada para todos los pacientes que requieren servicios intensivos, la asistencia parcial aguda podría ser una alternativa a la hospitalización completa o simplemente un complemento para acortar la duración de ésta y, por tanto, mejorar la satisfacción, la adaptación social y reducir los costes [2].

Pocos estudios han medido la evolución después del alta del tratamiento parcial agudo. Por tanto, hemos llevado a cabo una investigación sobre la evolución después de hospitalización parcial. El propósito del presente estudio era evaluar el tiempo hasta la recuperación de la masa corporal en estas pacientes.

Incluimos a las 27 pacientes con trastorno de la conducta alimentaria ( $88 \%$ de pacientes con anorexia nerviosa) hospitalizadas en 1999 en la unidad de asistencia parcial de un hospital general. Su IMC se evaluó en el episodio índice, en el alta y también después de un máximo de 2 años de seguimiento como pacientes ambulatorias de un programa de tratamiento intensivo. El tratamiento incluía psicoterapia de orientación cognitivo-conductual, la implicación de las familias en la terapia y el apoyo de la autoayuda.

La edad media de la muestra era 20,59 años (DT = $5,22)$. La media de días de hospitalización era 18,37 (DT =9,29). Casi el 50\% tenía en la inclusión un IMC $<17,5 \mathrm{~kg} / \mathrm{m}^{2}$. La prueba de la $t$ de Student para datos emparejados mostró una mejoría media de $0,9 \mathrm{~kg} / \mathrm{m}^{2}$ (IC del 95\%: 0,65-1,17 kg/m²) en el alta. Todas las pacientes con IMC $>17,5 \mathrm{~kg} / \mathrm{m}^{2}$ en la línea de base y $1 / 3$ de las pacientes con una masa corporal inferior en la admisión tenían IMC mayores que $17,5 \mathrm{~kg} / \mathrm{m}^{2}$ en el alta, y mantuvieron su mejoría durante un seguimiento medio de 62,26 días (DT $=61,13$ ). Además, el análisis de supervivencia mostró que las pacientes que no alcanzaron una masa de peso corporal superior a 17,5 durante la hospitalización tenían una probabilidad del $50 \%$ de alcanzarla en los 24 días posteriores al alta y una probabilidad del $72 \%$ de alcanzarla en los 36 días posteriores al ella. No hubo abandonos del estudio.

Los presentes hallazgos muestran que la hospitalización parcial no sólo es beneficiosa para la recuperación de las pacientes durante la hospitalización, sino que la mejoría continúa durante los días posteriores al alta. La hospitalización parcial en los hospitales generales es especialmente útil para estas pacientes, pues estas instituciones tienen el nivel apropiado de personal, programas de tratamiento

Rodriguez C, Fernández-Corres B, Pérez M-J, Iruin $\Lambda$, González-Pinto A. Partial hospitalization and outcome of anorexia nervosa. Eur Psychiatry 2002; 17: 236-237. 
muy estructurados y un acceso rápido y sencillo a la asistencia hospitalaria [2].

De modo interesante, se ha propuesto que la asistencia hospitalaria de la anorexia nerviosa se asocia con una evolución mala [3], pero esta afirmación bastante prematura podría llevar a la deducción de que el tratamiento hospitalario no se debería considerar [4]. La hospitalización y la hospitalización parcial son dos de los medios terapéuticos a nuestra disposición para tratar a estas pacientes. Concluimos que la hospitalización parcial se relaciona con la mejoría en nuestras pacientes con trastorno de la conducta alimentaria.

\section{BIBLIOGRAFÍA}

1 Gerlinghoff $M$, Backmund $H$, Franzen U, et al. Structured day care therapy program for eating disorders. Psychother Psychosom Med Psychol 1997;47:12-20.

2 Horvitz-Lennon M, Normand S, Gaccione P, et al. Partial versus full hospitalization for adults in psychiatric distress: a systematic review of the published literature (1957-1997). Am J Psychiatry $2001 ; 158: 676-85$.

3 Gowers SG, Wheetman J, Shore A, et al. Impact of hospitalization on the outcome of adolescent anorexia nervosa. $\mathrm{Br}$ J Psychiatry 2000;176:138-41.

4 Wood D, Flower P. Hospitalization and adolescent anorexia nervosa. Br J Psychiatry 2000;177:179. 